\title{
Actualización
}

\section{METODOLOGÍA PARA LA SELECCIÓN E INCORPORACIÓN DE ÁRBOLES PLUS EN PROGRAMAS DE MEJORAMIENTO GENÉTICO FORESTAL}

\author{
Jonathan Vallejos ${ }^{*}$,Yorleny Badilla*, Félix Picado**, Olman Murillo ${ }^{1 / *}$ \\ Palabras clave: Selección, árbol plus, mejoramiento genético, diferencial de selección, ganancia genética, \\ teca, Tectona grandis.
}

Keywords: Plus tree, tree breeding, selection differential, teak, Tectona grandis.

Recibido: 13/10/09

Aceptado: $15 / 03 / 10$

\section{RESUMEN}

Se presenta una metodología para evaluar y validar la superioridad de un árbol plus con base en su fenotipo, como parte de un programa de mejoramiento genético forestal. La metodología se basa en la evaluación fenotípica del árbol candidato y la comparación de su superioridad en relación con sus mejores vecinos en un radio inmediato de 10 a $15 \mathrm{~m}$. La evaluación permite crear un registro del árbol plus y validar la superioridad fenotípica del árbol según los caracteres volumen comercial y calidad. Se revisa y discute detalle relacionado con intensidad de selección (i) y número de árboles a seleccionar en una misma finca; caracteres a utilizar; la importancia de utilizar la yema dominante y no la bifurcación en especies latifoliadas; edad de selección; entre otros aspectos. Con el fin de evitar los errores de selección tipo I: individuos seleccionadosqueno son superiores genéticamente y tipo II: individuos no seleccionados, pero que si son superiores genéticamente. Se revisa el método de selección por comparación con los mejores 4 vecinos en su mismo microambiente. El árbol candidato se clasifica en 2 listas según su condición de superioridad fenotípica: lista A para el árbol que presenta superioridad en volumen y calidad, y lista B para ubicar el árbol que registra superioridad fenotípica únicamente en 1 de los 2 caracteres (volumen ó calidad). La metodología

1/ Autor para correspondencia. Correo electrónico: olmuga@yahoo.es

\section{ABSTRACT}

Plus trees selection and incorporation in tree improvement programs. A revised methodology for plus tree evaluation and superiority determination on the basic of its phenotypical attributes, as part of a breeding program, is presented. The methodology is based on a phenotypical evaluation of each candidate and its best four neighboring trees, in a 10 to $15 \mathrm{~m}$ radius. The field evaluation allows the establishment of a register for each plus tree candidate, with records for commercial volume and a joint criterium called tree quality. Aspects of intensity selection (i); number of trees selected per farm; age of selection; character selection; dominant bud as a better selection criteria in hardwoods, among others, are thoroughly revised. With the hope of avoiding type I selection errors (selected trees which are not truly genetically superior) and type II selection errors (trees that are truly genetically superior, but not selected), the selection method is revised by comparison with the best 4 neighboring trees, and by the establishment of 2 lists of candidate plus trees. List A is for these plus tree which was determined in field as superior in volume as well as in quality. List B is for these plus tree which may show some doubts, and found phenotypically superior only in volume or only in quality. This method allows the production of several statistics and

\footnotetext{
* Escuela de Ingeniería Forestal. Instituto Tecnológico de Costa Rica, Cartago, Costa Rica. BARCA S.A. Costa Rica.
} 
permite obtener un diferencial de selección, que se puede interpretar como la ganancia potencial a obtener al seleccionar y utilizar los mejores árboles por fenotipo. Se muestra un ejemplo real basado en uno de los programas de mejoramiento genético de teca (Tectona grandis) de una de las empresas miembro de la cooperativa de mejoramiento genético GENFORES.

\section{INTRODUCCIÓN}

La identificación y selección del árbol de alto rendimiento, es el inicio y la base fundamental de un programa de mejoramiento genético forestal. En relación con la calidad y rigurosidad con que se realice la selección de estos árboles, así será en concordancia la ganancia genética que se alcanzará. La rigurosidad en la selección se estima a través del concepto conocido como intensidad de selección “ $i$ ”; y se puede también expresar en términos de la magnitud del diferencial de selección "S", que se define de manera clásica como la distancia entre el promedio del conjunto de los individuos seleccionados y el promedio de la población original, para un caracter determinado (Zobel y Talbert 1984). Sin embargo, aún cuando se logre una selección estricta del material, poco se avanzará en el trabajo de mejoramiento si no hay una alta variabilidad genética en la población para el o los caracteres a mejorar (Resende et al. El método de selección del árbol plus y su incorporación temprana, Editorial Tecnológica de Costa Rica).

La mayor parte del conocimiento sobre el tema nace de los primeros programas de mejoramiento genético forestal, a finales de los años 50 con especies del género Pinus (Zobel y Talbert 1984). Por tanto, los criterios de selección para pinos no necesariamente coinciden con los utilizados en especies latifoliadas tropicales, con ciclos de vida mucho más cortos, la mayoría con una arquitectura de copa diferente. A pesar de interpretation possibilities. It helps in the initial definition of the commercial breeding population from the whole breeding population. An example based on real data from one of our teak (Tectona grandis) breeding programs, as part of the tree improvement cooperative GENFORES, is shown in detail.

la trascendencia del tema, pocos investigadores lo han abordado en los últimos años. Cornelius (1994) examinó 24 publicaciones sobre la efectividad de la selección de árboles plus en programas de mejoramiento genético donde registró, que la ganancia genética reportada y obtenida al utilizar material superior es mayor al $15 \%$ para los caracteres crecimiento en altura y crecimiento en diámetro (DAP), así como mayor a un $35 \%$ en volumen por unidad de área. En una plantación de Gmelina arborea en Campeche, sur de México, se registró a los 3 años de edad, un diferencial de selección de 3,8 m en altura y de 4,5 en diámetro (DAP) al escoger fenotípicamente los mejores 20 individuos (Balcorta y Vargas 2004).

Como concepto fundamental, debe recordarse que la selección de los árboles plus se realiza exclusivamente en plantaciones, con base en la expresión fenotípica de caracteres de interés. Por tanto, la manifestación de estos caracteres está siempre bajo efectos ambientales, efectos genéticos (dominancia y epistasis), que podrían ser muy altos en algunos casos y confundir al seleccionador con lo que observe fenotípicamente (Cruz 2005). De tal forma que el seleccionador no podrá distinguir entre un individuo genéticamente superior de otro que aparenta serlo. Para caracteres con un efecto de dominancia completa, no podrá diferenciar entre un homocigota y un heterocigota para los alelos deseables $\left(\mathrm{A}_{\mathrm{i}} \mathrm{A}_{\mathrm{i}}\right.$ de $\left.\mathrm{A}_{\mathrm{i}} \mathrm{A}_{\mathrm{j}}\right)$. Si la persona que selecciona y sanciona el árbol candidato no es calificada en la materia, podrán ocasionarse errores de selección graves. Estas situaciones 
provocan 2 tipos de error en los programas jóvenes de mejoramiento genético: error tipo I, que es cuando se seleccionan individuos que realmente no son superiores genéticamente; error tipo II, cuando en un programa no se incluyen individuos que si son genéticamente superiores al promedio poblacional. Simplemente no es fácil que puedan ser detectados fenotípicamente en campo. El error tipo I causa un aumento de materiales en la población base de mejoramiento, que deben ser evaluados en campo y elevarán los costos del programa. Si la base de ensayos genéticos es insuficiente, algunos de estos individuos permanecen por más tiempo en la población de mejoramiento sin merecerlo, al provocar disminuciones en el progreso genético realizado. Si el programa se basa en reproducción sexual, entonces se obtendrá una segregación mendeliana mayor y una proporción relativamente alta de fenotipos indeseables. El programa avanzará más lentamente. El error tipo II ocasiona una reducción potencial en las ganancias genéticas del programa, ya que genotipos de alto valor no llegarán nunca a formar parte de la población de mejoramiento. Con el fin de reducir ambos tipos de errores, en el Programa de Mejoramiento Genético Forestal desarrollado por la Escuela de Ingeniería Forestal del Instituto Tecnológico de Costa Rica, en conjunto con 10 empresas reforestadoras (GENFORES), se ha proporcionado un nuevo protocolo de selección y sanción fenotípica, más eficiente, objetivo y práctico, que se comparte en esta publicación.

Los efectos ambientales serán de tal magnitud en la expresión fenotípica, que la posibilidad de cometer errores tipo I ó tipo II será prácticamente la que predomine. Esta situación es típica en programas de mejoramiento de especies forestales nativas, que intentan aplicar los principios de selección de árboles plus en rodales naturales. Salvo en los casos de la mayoría de las especies coníferas y algunas pocas especies latifoliadas, que presentan ya evolutivamente una alta dominancia apical, no será eficiente utilizar estos procedimientos. En condiciones naturales es poco probable que al seleccionar un árbol se tenga la certeza de la superioridad genética en crecimiento o alta producción de volumen. En estos casos se utiliza el concepto de árbol semillero, que a pesar de que también se revisa que posea buenas características fenotípicas en el árbol seleccionado, no se le debe atribuir en esta etapa ningún calificativo de superioridad genética. En este tipo de programas será más importante una buena base genética, de modo que se debe colectar y reproducir por separado la semilla de cada árbol. Los árboles se seleccionan separados al menos por $100 \mathrm{~m}$ de distancia uno de otro, a fin de establecer posteriormente ensayos de progenie, donde sí se podrá entonces en condiciones de plantación, realizar una selección eficiente y certera (Murillo et al. 2001, Badilla et al. 2002).

\section{MATERIALES Y MÉTODOS}

\section{Evaluación de árboles potenciales y su fundamentación}

A partir del año 2001 se inicia en Costa Rica un programa de mejoramiento genético forestal cooperativo y vinculado entre la Escuela de Ingeniería Forestal del Instituto Tecnológico de Costa Rica y un grupo de empresas reforestadoras denominado GENFORES, que fue extendiéndose luego a Colombia, hasta incluir hoy día a 10 empresas miembro (Gutiérrez et al. 2003). Con base en el proceso de selección e incorporación a la fecha de más de 900 árboles, plus de teca (Tectona grandis), melina (Gmelina arborea), acacia (Acacia mangium), pilón (Hyeronima alchorneoides), amarillón (Terminalia amazonia) y otras especies (Murillo et al. 2004), se consolidó un protocolo de trabajo, mejorado a través de los años.

\section{La evaluación del árbol potencial en relación con sus mejores vecinos}

El proceso de selección inicia con una primera revisión de toda la plantación, se buscan individuos sobresalientes en crecimiento o en calidad de su fuste, como se describirá más adelante. Las plantaciones deben haber superado 
fenotípica evidente o en el volumen de crecimiento o en su calidad para la producción de madera de alto valor industrial. Este procedimiento permite garantizar la superioridad genética de los individuos seleccionados en ese ambiente; sin embargo, no podrá garantizar su desempeño en un ambiente diferente, para lo cual son necesarios los ensayos de validación.

Se debe recordar como principio, que en la mayoría de los casos, los caracteres que determinan el volumen actúan por separado de los que controlan la calidad. Ambos grupos de caracteres son de importancia en un programa de mejoramiento genético. Pocos individuos en la naturaleza son superiores en todos sus caracteres. Por tanto, algunos individuos portadores de buenas características cualitativas podrían ser de interés para ser luego cruzados en forma controlada con individuos de superioridad volumétrica. Esto implica, que algunos árboles encontrados en campo, podrían ser de interés dentro de la llamada población de mejoramiento genético, donde se evalúan y cruzan para producir las próximas generaciones de mejoramiento. Este procedimiento permite que sean los ensayos genéticos, en condiciones experimentalmente controladas los que determinen, con base en el valor genético, cuáles individuos seleccionados como plus son verdaderamente superiores genéticamente, denominados entonces como árboles élite.

\section{Caracteres que deben ser evaluados en la selección del árbol plus}

Como principio, todo carácter debe tener: a) un alto control genético (heredabilidad), b) alta variación genética, c) preferiblemente de fácil medición (Zobel y Talbert 1984). La discusión más importante gira en torno a cuáles caracteres debe dársele más peso en la decisión final al calificar un árbol candidato como árbol plus. Debe tenerse presente que a pesar de que se selecciona el árbol como individuo, la unidad base del programa de mejoramiento genético y la decisión de su elección dependerá de la expresión de uno o varios caracteres o atributos sobresalientes. Hoy día podemos de manera simplificada agrupar las plantaciones forestales en 3 grandes tipos con objetivos y manejo diferentes:

1. Arboricultura: paisajismo, restauración y otros.

2. Plantaciones para la producción máxima de biomasa por unidad de área (producción de fibra, carbón o energía).

3. Plantaciones para la producción de madera sólida de alta calidad y mayor volumen por árbol.

Los caracteres evaluados en un árbol plus, son los de mayor retribución económica.

En el caso de la arboricultura se incluye el paisajismo, arborización de fincas y ciudades, estabilización de suelos, restauración de ambientes, entre otros. En cuyo caso se privilegian caracteres como: a) capacidad de floración; b) forma de copa; c) capacidad de crecer en suelos marginales; d) tolerancia a ambientes contaminados; e) tolerancia al viento; f) sanidad, g) vigor; entre otros (Torres y Magaña 2001).

Para la producción de biomasa, interesa esencialmente evaluar caracteres como: a) una alta sobrevivencia; b) mayor altura comercial; c) rectitud del fuste y d) esencialmente capacidad de crecimiento bajo densidades altas. Sus características cualitativas tienen un peso menor, aunque el fuste recto será siempre un caracter a incluir. En este tipo de plantaciones lo que se privilegia es la producción de volumen por unidad de área.

Para la producción de madera de alta calidad y volumen por árbol individual, se privilegian árboles con características cualitativas de fuste más exigentes. Se elegirán árboles capaces de acumular mayor volumen individual y con alta dominancia apical como principal criterio de selección. Es decir, el ideotipo del árbol plus es aquel que invierte la mayor parte de su energía en el fuste y poco en ramas. Una mayor cantidad de caracteres deberán ser por tanto considerados en el momento de sancionar el árbol candidato a plus, como se mencionará adelante. Una variante de este tipo de plantaciones productivas son los 
sistemas agroforestales, donde son importantes caracteres como: a) menor tamaño de copa; b) menor cantidad de ramas por metro de fuste; c) menor conicidad del fuste en espacios abiertos y d) menor grosor de ramas.

Se ha presentado con frecuencia la intención de mejorar especies nativas de alto valor, donde ocurre en muchos casos que sus hábitos de crecimiento en el nuevo ambiente de plantación, impide obtener un fuste comercial. En estos casos, la mayor altura comercial posible se convierte en el carácter más importante. Ejemplos de estos casos son las experiencias con las especies de los géneros Tabebuia, Dalbergia, Enterolobium, la especie Pithecelobium saman y varios géneros de la familia Meliaceae.

Por lo general se trabaja con caracteres cuantitativos o relacionados con un mayor volumen del árbol: a) diámetro a la altura del pecho; b) altura total; c) mayor altura comercial o punto donde pierde la dominancia apical. Así como caracteres cualitativos, más importantes que aseguren una mayor calidad del fuste para la producción de madera sólida que son por lo general: a) rectitud del fuste, b) alta dominancia apical, c) inserción de ramas al fuste lo más cercano al ángulo recto, d) ramas delgadas, e) sin bifurcación, f) sin ramas en reiteración, g) sin grano en espiral, h) sano y libre de enfermedades, i) vigoroso, j) sin gambas o aletones basales, k) sin cola de zorro (en Pinus spp).

\section{Caracteres cuantitativos o aditivos}

Los caracteres asociados al volumen del fuste registran normalmente una baja heredabilidad (control genético), debido a que el ambiente de la plantación como densidad de siembra y competencia entre arboles, influye en gran medida en su desarrollo. Un caracter como el crecimiento de un árbol se estima está controlado por una gran cantidad de loci que involucra una porción muy alta del genoma. Los valores de heredabilidad de estos caracteres raramente superan un $40 \%$ $\left(h^{2} \leq 0,4\right)$. Como caso dramático está el diámetro a la altura del pecho, que es altamente sensible a la competencia y reporta por lo general, valores de heredabilidad sumamente bajos $\left(\mathrm{h}^{2}<0,25\right.$ a 0,35 ; Cornelius 1994), en particular si la selección se realiza en plantaciones y no en ensayos genéticos. Por el contrario, la altura total del árbol tiene un efecto menor de la competencia o ambiente de crecimiento. Dado que todo programa busca prioritariamente una mayor producción de volumen por árbol, se selecciona aquellos individuos con base en a) su superioridad en altura total y se utiliza en menor grado el criterio del b) diámetro a la altura del pecho. Puede entonces perfectamente elegirse a un individuo con base en su superioridad en altura, en relación con sus mejores 4 ó 5 vecinos, a pesar de que su DAP no los supere. De esta manera se elige indirectamente aquellos individuos superiores en volumen, con base en un carácter de mayor control genético. Esta decisión permite disminuir el error tipo I, que típicamente ocurre cuando se eligen árboles únicamente por su buen crecimiento en diámetro, cuya superioridad no se vuelve a repetir al ser plantado en otras condiciones ambientales. De modo que se elijan los individuos capaces de producir mayor volumen por árbol. Puede observarse en el Cuadro 1 la columna que registra el número de vecinos presentes alrededor del candidato de los 8 posibles. Este criterio puede ser de utilidad en caso de duda sobre el vigor y crecimiento potencial del candidato. Puede ser utilizada para desempatar cuando algún vecino iguale en DAP al árbol candidato. El DAP del individuo con mayor cantidad de vecinos (competencia) es el de mayor mérito.

\section{Caracteres cualitativos}

Los caracteres cualitativos registran con frecuencia una mayor heredabilidad, debido a que se estima están controlados por un número reducido de loci (por lo general $\mathrm{h}^{2}>0,5$ ) y están sometidos a una menor presión del ambiente. Esto implica que un árbol superior a sus vecinos en algún carácter cualitativo, por ejemplo ausencia de grano en espiral, mantendrá esa superioridad en prácticamente cualquier ambiente en que se le plante.

En años recientes se ha desarrollado en el país una metodología de evaluación de la calidad 
de los árboles, que integra todos los caracteres cualitativos individuales en uno solo colectivo denominado calidad (Murillo y Badilla 2004). Se califica individualmente cada troza de $2,5 \mathrm{~m}$ de largo según sus atributos cualitativos para la producción de madera sólida (Figura 1). La calificación se hace con un valor de 1 a 4, donde un valor de 1 significa excelente en todos los caracteres; un valor de 2 significa que se observaron defectos menores en uno o varios caracteres como presencia de ramas gruesas, ángulo muy agudo de inserción de ramas al fuste, presencia de gambas pequeñas, rectitud levemente torcida, entre otros; se califica con un 3 cuando la troza registra defectos visibles y severos en varios caracteres que no permiten su utilización industrial en más de un 50\%. Como ejemplo está un daño causado por alguna plaga o una torcedura severa en el fuste. Finalmente se califica con un valor de 4 trozas sin ninguna posibilidad de aserrío, por ejemplo, con torceduras excesivas, presencia de grano en espiral, bifurcada o dañada completamente por alguna plaga. Se continúa sucesivamente calificando una a una las siguientes trozas de 2,5 $\mathrm{m}$ de longitud, hasta alcanzar los $10 \mathrm{~m}$ de altura del fuste o primeras 4 trozas de $2,5 \mathrm{~m}$. Esta calificación se realiza simultáneamente a los 4 ó 5 mejores vecinos del árbol candidato.

Con esta calificación individual de las primeras 4 trozas en cada árbol, se obtiene luego en oficina una nueva variable que se denomina calidad del árbol, que se calcula como sigue:

Calidad del árbol $=$ calidad troza $1 * 0,4+$ calidad $\mathrm{t} 2 * 0,3+$ calidad $\mathrm{t} 3 * 0,2+$ calidad $\mathrm{t} 4 * 0,1$ (1).

Los pesos asignados a cada troza corresponden con su aporte individual al volumen total del árbol.

\section{Bifurcación y la yema apical dominante}

La presencia de una bifurcación ha sido típicamente utilizada como criterio de eliminación de un árbol candidato a árbol plus y reportado como un carácter con un alto control genético (Zobel y Talbert 1984). Sin embargo, en especies latifoliadas, con una arquitectura de copa muy diferente, es común encontrar individuos que

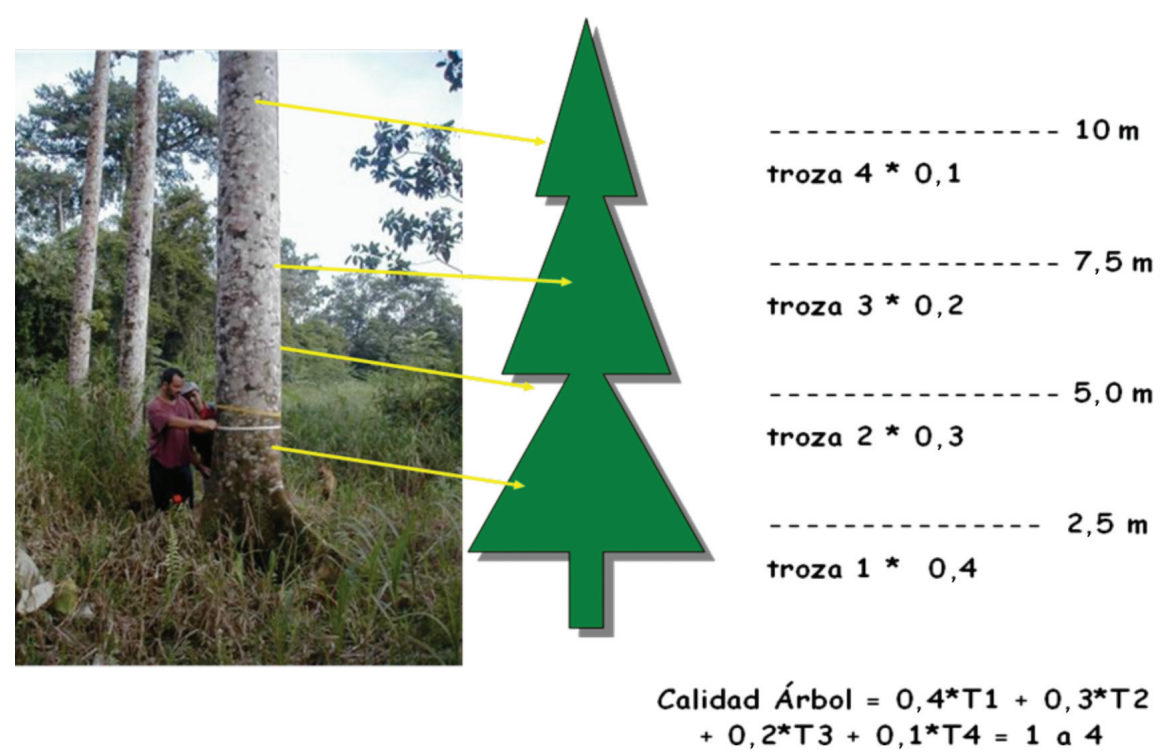

Fig.1. Evaluación fenotípica de la calidad de un árbol candidato a árbol plus, con base en la calificación de la calidad de sus primeras 4 trozas de $2,5 \mathrm{~m}$ de largo cada una. 
inician la formación de copa a muy baja altura, o especies con alta fragilidad a la pérdida de su meristemo principal por efecto del viento, plagas, deficiencia de nutrimentos menores (boro, zinc o cobre en Eucalyptus spp). Todos estos casos ocasionarán la presencia de una bifurcación a baja altura, justificada totalmente por efectos ambientales. Un seleccionador poco experimentado podría fácilmente cometer el error tipo II, al descalificar árboles candidato que podrían ser genotípicamente superiores.

Un árbol con una clara dominancia apical y vigoroso tiene una mayor probabilidad de no presentar bifurcaciones, pero también, la de no permitir la presencia de ramas gruesas, o ramas que compitan con el eje dominante denominadas reiteraciones. Este tipo de árboles invierten una mayor cantidad de energía en su fuste principal, que evita la desviación de energía hacia las ramas. Estos individuos son los que registran luego la mayor altura comercial y se han convertido en uno de los caracteres de mayor importancia durante la sanción de los árboles candidato en especies latifoliadas.

\section{Selección de árboles en los sitios}

Si se logra encontrar algún individuo sobresaliente en un sitio sumamente marginal, es muy probable que se trate de un genotipo de alto potencial. Varias experiencias positivas se han obtenido con esta práctica. Individuos capaces de crecer en condiciones de sitio muy adversas, luego en sitios bien preparados y fertilizados logran un alto desempeño. La explicación está posiblemente relacionada con individuos, cuyo genoma permite que sean fisiológicamente más eficientes. Son genotipos que logran crecer y producir madera aún bajo alguna de las siguientes condiciones que por lo general, son las que explican en gran proporción la baja productividad de plantaciones forestales: baja fertilidad, alta saturación de acidez, alta compactación del suelo, baja precipitación, periodo seco prolongado, baja profundidad efectiva del suelo, entre los más relevantes.

\section{Árboles que deben ser seleccionados en una finca}

Un programa de mejoramiento genético debe basar su población de mejoramiento en unos 200 a 300 genotipos si pretende continuar la obtención de ganancias genéticas a largo plazo (Zobel y Talbert 1984, Murillo 1991). Sin embargo, para alcanzar este tamaño de población y evaluar en campo correctamente todos los individuos seleccionados, se requiere de muchos recursos, lo cual imposibilita que una organización pequeña pueda en forma aislada pretender desarrollar un programa de este tipo. Basado en este principio se han conformado modelos asociativos o cooperativos en varios países, para poder atender el desarrollo apropiado de programas de mejoramiento genético forestal (Gutiérrez et al. 2003). Por lo general en nuestro medio, un buen programa para una empresa logra seleccionar y evaluar adecuadamente unos 50 a 70 genotipos, según experiencia por más de 8 años de los investigadores.

Pero si se aborda la pregunta desde el punto de vista de la intensidad de selección (i), la experiencia generada en GENFORES en estos 9 años ha sido, en promedio, seleccionar 1 árbol plus por cada 15000 a 20000 individuos, que equivale aproximadamente a 1 árbol cada 15 a 20 ha. En casos críticos se ha logrado seleccionar un árbol cada 100 ha, y dependerá siempre de la disponibilidad real de plantaciones donde seleccionar. Debe recordarse que si se utilizan criterios muy estrictos, se corre el riesgo de cometer el error tipo II, que consiste en dejar por fuera del programa excelentes genotipos que los efectos ambientales o de dominancia pudieran ocultar; sin embargo en algunas ocasiones se eligen individuos que muestren un fuste con algunos defectos leves como torcedura leve o ángulo de ramas agudo, pero que manifiestan un crecimiento y desarrollo volumétrico excepcional. Así también se seleccionan a veces individuos, que a pesar de tener un diámetro a la altura pecho menor a sus vecinos, su altura total alcanza el dosel superior (dominante) y la calidad de su fuste es excepcionalmente buena para la producción maderera (Murillo et al 2003). 
Este criterio no debe asumirse en forma rígida, sino como una referencia. No pocas veces se revisa una finca completa donde no se encuentra un solo individuo tan siquiera aceptable.

Los árboles seleccionados deben estar bien identificados y georeferenciados en el campo, con el fin de facilitar los procesos de evaluación y de recolección de material vegetativo para su propagación e incorporación al programa de mejoramiento.

En el Cuadro 1 se presenta el formulario de campo utilizado para evaluar el fenotipo del árbol candidato y de los vecinos. Con la información aportada se logra obtener un registro del árbol plus y permite validar la superioridad del árbol plus con respecto a sus mejores vecinos.

Una vez que se ha evaluado los árboles en el campo se estima el volumen comercial de cada individuo con base en su Dap y altura comercial. La calidad general del árbol se obtiene del promedio de la calidad de sus trozas individuales, con una ponderación de cada troza según su posición dentro del fuste (Figura 1). La variable calidad del árbol genera valores entre 1 y 4, donde la mejor calidad tiene un valor de 1 .

\section{Edad de selección}

Debe aclararse que la selección del árbol plus, en programas nuevos de mejoramiento genético es totalmente fenotípica y ocurre en plantaciones ya establecidas, por tanto, bajo condiciones ambientales no controladas. Diferente será la situación de selección que se presenta en programas avanzados de mejoramiento genético, donde los árboles élite son seleccionados en ensayos genéticos y los criterios de selección se fundamentan casi exclusivamente en sus valores genéticos (Resende et al.)

La edad de selección es fundamental en todo programa joven de mejoramiento genético. El técnico pretende encontrar individuos superiores genéticamente en una serie de caracteres, que en muchos casos no se han expresado completamente a edades tempranas. Esta situación es particularmente difícil con caracteres cualitativos como fibra (grano) en espiral, aparición de gambas o aletones basales, hábitos de cicatrización de ramas, grado de dominancia apical y algunos defectos de la forma del fuste, que por lo general se pueden observar claramente hasta aproximadamente los 5 ó 6 años de edad para especies en zonas tropicales. Así también, la susceptibilidad extrema a algún patógeno como por ejemplo Fusarium en Tectona grandis, no será posible determinarla antes de unos 7 años de la plantación. Algunas propiedades de la madera, como el peso específico y la aparición del duramen, son esenciales para la selección de los árboles plus, caracteres que difícilmente podrán ser evaluados antes de los 4 a 5 años de edad.

De manera quizá compleja será el poder determinar, de manera objetiva, la superioridad en crecimiento en individuos jóvenes con una competencia por vecinos desigual en cada caso. El diámetro del árbol es uno de los caracteres bajo mayor efecto ambiental (competencia), por tanto con valores bajos de heredabilidad en la mayoría de los casos (Cornelius 1994), lo que implica que el diámetro es un caracter riesgoso para ser utilizado en la selección fenotípica temprana de árboles plus. Sin embargo, un árbol con un excelente diámetro muestra alto potencial o vigor. Significa esto, que si se logran reproducir las condiciones ambientales donde crece, podrá repetir ese extraordinario crecimiento. Por tanto, a pesar de ser cierto lo de su baja heredabilidad y mayor efecto ambiental, no debe dudarse en incluir individuos con un crecimiento diamétrico extraordinario. Una posibilidad de reducir el error de selección (tipo I), es el de incluir el nivel de competencia que presentan los árboles candidato (Cuadro 1). Para esto simplemente se contabiliza cuántos individuos de los 8 posibles están presentes en la parcela imaginaria alrededor suyo. Se interpreta que tendrá mayor mérito aquel individuo que exprese un DAP superior, a pesar de crecer rodeado de todos sus vecinos.

En caso de que el árbol candidato sea muy semejante en diámetro a sus vecinos, la altura total, la altura comercial o la dominancia apical, son mejores criterios de selección en árboles jóvenes. Debe recordarse que la altura del árbol es un carácter bajo 
menor efecto de la densidad o competencia de plantación. Por tanto, un buen indicador de superioridad en volumen y crecimiento del individuo. Dadas estas condiciones, se ha observado que la selección antes de los 5 ó 6 años de edad implica un alto riesgo de cometer el error tipo I, que es la incorporación de muchos individuos que no son genéticamente superiores para los caracteres de interés.

De manera también importante es tener algún cuidado con la selección de árboles plus en plantaciones de mucha edad. El riesgo principal que se ha observado es la imposibilidad de detectar caracteres importantes, que se expresan a temprana edad. Este es el caso típico de la tendencia a formar gran cantidad de ramas por fuste lineal, así como la tendencia a formar ramas gruesas. En plantaciones viejas y bien manejadas, los programas de podas han eliminado las ramas en los primeros 5 a $7 \mathrm{~m}$ de fuste y hace imposible revisar estos caracteres. Sin embargo, puede considerarse que estos caracteres son de menor grado de prioridad en un programa de mejoramiento y que pueden luego fácilmente ser considerados en la siguiente generación de mejoramiento.

Por tanto, el criterio de selección de árboles plus después de los 6 años de edad es una adecuada edad mínima de referencia y que reduce las posibilidades de error tipo I y II de selección en programas jóvenes de mejoramiento genético.

En programas avanzados de mejoramiento podrá reducirse la edad de selección, dado que los materiales tienden a ser más homogéneos (homocigotas) producto del proceso de selección en cada generación de mejoramiento. Los análisis genético-cuantitativos y estadísticos tienden a ser más precisos y las correlaciones genéticas juvenil-adulto tienden a permitir obtener estimados certeros a temprana edad.

\section{RESULTADOS Y DISCUSIÓN}

\section{Validación de la superioridad fenotípica}

Para validar la superioridad fenotípica del árbol candidato con respecto a sus mejores vecinos, se analizan los 2 criterios fundamentales: los caracteres asociados al volumen y los asociados con la calidad del árbol (Vallejos 2007). El volumen integra la relación diámetro y altura comercial o yema apical dominante, mientras que la calidad del árbol integra todas las otras variables cualitativas: diámetro de ramas, ángulo de inserción de ramas, presencia de gambas o aletones, de grano en espiral, sanidad, etc. (Cuadro 1). Un árbol plus debe presentar características mínimas deseables como posición sociológica dominante, fuste muy recto, con ramas delgadas (grosor menor a $4 \mathrm{~cm}$ de diámetro $0<1 / 3$ del DAP), ángulo de rama entre $45^{\circ}$ a $90^{\circ}$, excelente estado fitosanitario, ausencia de gambas o aletones, ausencia de grano en espiral y por supuesto, la mayor altura comercial posible. El árbol plus debe superar a todos sus mejores vecinos en la parcela de comparación. Su superioridad debe ser con respecto a cada uno de sus vecinos individuales, y no tan solo con relación al promedio de sus vecinos. Si uno de los árboles vecinos supera al candidato en alguno de los caracteres de interés, entonces el vecino se convertirá en el nuevo candidato.

Para efectos estadísticos, de cada árbol candidato se obtiene el porcentaje de superioridad en volumen comercial y calidad con respecto a la media de los mejores 4-5 mejores vecinos. La transformación porcentual de cada variable se obtiene por medio de la siguiente expresión:

Superioridad $(\%)=\frac{\text { árbol plus }- \text { media de vecinos }}{\text { media de vecinos }} * 100$

Los árboles evaluados se clasifican en 2 listas: aquellos que presentan superioridad marcada en volumen y en calidad con relación a todos sus mejores vecinos individuales (árboles plus de la lista A). De este grupo, los mejores 15 a 20 individuos conformarán la población comercial. Mientras que aquellos individuos que no superen a alguno de sus vecinos en alguna de las 2 variables, se clasifican como árbol de la lista $\mathrm{B}$. Se asume que poseen al menos un carácter deseable, que podría ser capturado a través de cruzas controladas en el programa de mejoramiento. Los árboles de la lista B son individuos que merecen 
ser incorporados al programa de mejoramiento para evitar error tipo II. El argumento es que las dudas sobre su posible superioridad podrán ser mejor dilucidadas en un buen ensayo genético. Estos individuos no se incorporan a la población comercial, sino que forman parte de la población de mejoramiento y se mantienen a la espera de su evaluación genética y su utilización en los cruzamientos controlados.

En el Cuadro 2 se muestra el resultado de la evaluación de 26 árboles plus con respecto a sus mejores 4 vecinos. Los árboles plus fueron seleccionados en plantaciones de teca (Tectona grandis) de 9 a 12 años de edad, en el Pacífico Central de Costa Rica. Puede notarse que únicamente 10 árboles son potenciales para usar a escala comercial (lista A). En estos casos se debe seleccionar más árboles plus para evitar problemas ligados a una base genética, o también, intercambiar material genético con otras organizaciones. Dado que la superioridad fenotípica es expresada en porcentaje respecto a sus mejores vecinos, este valor puede ser utilizado para organizar un ranking fenotípico y poder decidir objetivamente, cuáles son los mejores 15 a 20 individuos que conformarán la población comercial, tal y como se muestra en el Cuadro 3.

La utilización de estos árboles plus de teca como clones, registra un diferencial de selección de un $22,9 \%$ en volumen comercial y un $21,8 \%$ en calidad. Si se utiliza únicamente los árboles plus de la lista A, el diferencial aumenta a un $35,5 \%$ en volumen comercial y un $33,7 \%$ en calidad. $\mathrm{Si}$ a los árboles plus de la lista $\mathrm{A}$ se le incorporan aquellos individuos de la lista B que registraron superioridad en volumen, el diferencial de selección aumentaría a un $46,7 \%$ en volumen comercial, pero su calidad aumentaría tan solo en un $14,2 \%$.

Un buen ejemplo de un árbol interesante para un programa de mejoramiento genético, es el individuo $\mathrm{N}^{\circ}$. 15, categorizado correctamente en la lista $\mathrm{B}$ (Cuadro 2). Este árbol registró poco más de $0,81 \mathrm{~m}^{3}$ de madera comercial, que supera a sus vecinos en más de un $210 \%$. Pero su calidad de fuste resultó ser baja. El árbol 26 muestra el caso exactamente al contrario. Una calidad de fuste extraordinaria, que supera en más de un $85 \%$ a sus vecinos; pero un crecimiento en volumen mediocre, que registra inclusive una inferioridad de poco más de un $20 \%$ con respecto a sus vecinos. Estos 2 casos serían claramente eliminados de un programa convencional de mejoramiento genético. Sin embargo, uno de ellos exhibe un extraordinario vigor y en el otro, una sobresaliente calidad de fuste, ambos criterios de importancia capital y altamente deseables. No debe olvidarse, que en la mayoría de los casos los caracteres de volumen y los cualitativos se segregan de manera independiente. El árbol ideal, superior en volumen y en calidad, será muy difícil de encontrar, pero es posible construirlo a través de cruzamientos controlados con progenitores sobresalientes. Una buena decisión es por tanto, incluirlos en la lista B, con el fin de poder determinar posteriormente, si esos atributos altamente deseables son heredables y por tanto, utilizables en el programa de mejoramiento. Sus atributos son tan sobresalientes, que podríamos cometer un grave error al no incluirlos en el programa de mejoramiento (error de selección tipo II).

\section{Estimación de la ganancia genética esperada}

Con la información de la superioridad fenotípica de los árboles plus puede estimarse una ganancia genética esperada. Debe tenerse presente que estos valores de diferencial de selección son por lo general más bajos de lo real, debido a que cada árbol plus fue evaluado contra sus mejores 4 vecinos. El argumento es que estos vecinos son competidores muy fuertes, y por tanto, no representativos del material ordinario (sin mejoramiento) que usualmente se obtiene de los viveros comerciales. Por lo tanto, el diferencial de selección registrado con este procedimiento, puede considerarse como conservador. Si se asume que estos árboles plus serán clonados directamente, y se multiplicarán centenares de veces para lograr un impacto inmediato en la calidad y volumen.ha- ${ }^{-1}$ de las plantaciones, 
Cuadro 2. Resultado de la validación de superioridad fenotípica en volumen comercial y calidad de los árboles plus de teca (Tectona grandis) en el Pacífico central de Costa Rica. 2008.

\begin{tabular}{|c|c|c|c|c|c|c|c|}
\hline \multirow{2}{*}{ Árbol plus } & \multirow{2}{*}{ Clasificación } & \multicolumn{2}{|c|}{ Árbol plus a) } & \multicolumn{2}{|c|}{ Mejores testigos b) } & \multicolumn{2}{|c|}{ Diferencial c) } \\
\hline & & Volumen $\left(\mathrm{m}^{3}\right)$ & Calidad & Volumen $\left(\mathrm{m}^{3}\right)$ & Calidad & Volumen $(\%)$ & Calidad (\%) \\
\hline 1 & B & 0,39 & 70 & 0,27 & 75 & 44,44 & $-6,67$ \\
\hline 2 & A & 0,41 & 100 & 0,33 & 67 & 24,24 & 48,51 \\
\hline 3 & $\mathrm{~B}$ & 0,31 & 97 & 0,37 & 73 & $-16,22$ & 31,82 \\
\hline 4 & $\mathrm{~B}$ & 0,31 & 87 & 0,33 & 75 & $-6,06$ & 15,56 \\
\hline 5 & A & 0,28 & 100 & 0,25 & 65 & 12,00 & 53,85 \\
\hline 6 & A & 0,25 & 97 & 0,22 & 67 & 14,09 & 43,56 \\
\hline 7 & $\mathrm{~B}$ & 0,08 & 100 & 0,2 & 90 & $-60,00$ & 11,11 \\
\hline 8 & B & 0,19 & 100 & 0,3 & 74 & $-36,67$ & 35,14 \\
\hline 9 & $\mathrm{~B}$ & 0,19 & 100 & 0,3 & 74 & $-36,67$ & 35,14 \\
\hline 10 & $\mathrm{~B}$ & 0,17 & 77 & 0,16 & 81 & 6,25 & $-4,96$ \\
\hline 11 & A & 0,30 & 77 & 0,29 & 62 & 3,13 & 24,32 \\
\hline 12 & $\mathrm{~B}$ & 0,34 & 77 & 0,2 & 81 & 69,50 & $-4,96$ \\
\hline 13 & B & 0,23 & 97 & 0,24 & 69 & $-4,17$ & 40,10 \\
\hline 14 & $\mathrm{~B}$ & 0,31 & 90 & 0,24 & 92 & 29,17 & $-1,82$ \\
\hline 15 & B & 0,81 & 33 & 0,26 & 68 & 211,54 & $-51,22$ \\
\hline 16 & B & 0,41 & 50 & 0,23 & 77 & 78,26 & $-34,78$ \\
\hline 17 & A & 0,22 & 100 & 0,21 & 67 & 4,76 & 48,51 \\
\hline 18 & $\mathrm{~B}$ & 0,16 & 87 & 0,16 & 79 & 0,00 & 9,70 \\
\hline 19 & $\mathrm{~B}$ & 0,29 & 97 & 0,30 & 84 & $-3,33$ & 15,08 \\
\hline 20 & $\mathrm{~B}$ & 0,27 & 100 & 0,32 & 64 & $-15,63$ & 56,25 \\
\hline 21 & A & 0,57 & 97 & 0,47 & 70 & 21,28 & 38,10 \\
\hline 22 & A & 0,78 & 100 & 0,40 & 81 & 95,00 & 23,97 \\
\hline 23 & A & 0,33 & 100 & 0,20 & 76 & 65,00 & 32,16 \\
\hline 24 & A & 0,38 & 100 & 0,29 & 83 & 31,03 & 20,00 \\
\hline 25 & A & 0,59 & 87 & 0,32 & 83 & 84,38 & 4,00 \\
\hline 26 & $\mathrm{~B}$ & 0,39 & 100 & 0,49 & $\underline{54}$ & $-20,41$ & 85,19 \\
\hline
\end{tabular}
a) Diferencial de selección de todos los árboles plus=22,88\% (volumen) y 21,83\% (Calidad).
b) Diferencial de selección únicamente de los árboles plus A=35,49\% (Volumen) y 33,7\% (Calidad).
c) Diferencial de selección de todos los árboles plus superiores en volumen=46,71\% y 14,2\% (Calidad). 
Cuadro 3. Ranking fenotípico de los árboles plus de la lista A de teca (Tectona grandis), seleccionados en el pacífico central de Costa Rica organizados por su mayor diferencial de selección en volumen. 2008.

\begin{tabular}{|c|c|c|c|c|c|c|c|}
\hline \multirow[b]{2}{*}{ Árbol Plus } & \multirow[b]{2}{*}{ Clasificación } & \multicolumn{2}{|c|}{ Árbol Plus } & \multicolumn{2}{|c|}{ Mejores Testigos } & \multicolumn{2}{|c|}{ Diferencial } \\
\hline & & $\begin{array}{c}\text { Volumen } \\
\left(\mathrm{m}^{3}\right)\end{array}$ & Calidad & $\begin{array}{l}\text { Volumen } \\
\left(\mathrm{m}^{3}\right)\end{array}$ & Calidad & $\begin{array}{l}\text { Volumen } \\
(\%)\end{array}$ & $\begin{array}{c}\text { Calidad } \\
(\%)\end{array}$ \\
\hline 22 & A & 0,78 & 100 & 0,4 & 81 & 95,00 & 23,97 \\
\hline 25 & A & 0,59 & 87 & 0,32 & 83 & 84,38 & 4,00 \\
\hline 23 & A & 0,33 & 100 & 0,20 & 76 & 65,00 & 32,16 \\
\hline 24 & A & 0,38 & 100 & 0,29 & 83 & 31,03 & 20,00 \\
\hline 2 & A & 0,41 & 100 & 0,33 & 67 & 24,24 & 48,51 \\
\hline 21 & A & 0,57 & 97 & 0,47 & 70 & 21,28 & 38,10 \\
\hline 6 & A & 0,25 & 97 & 0,22 & 67 & 14,09 & 43,56 \\
\hline 5 & A & 0,28 & 100 & 0,25 & 65 & 12,00 & 53,85 \\
\hline 17 & A & 0,22 & 100 & 0,21 & 67 & 4,76 & 48,51 \\
\hline 11 & A & 0,30 & 77 & 0,29 & 62 & 3,13 & 24,32 \\
\hline
\end{tabular}

entonces la ganancia genética esperada podría estimarse como sigue:

$\mathrm{GG}=\mathrm{S}$ (diferencial de selección) $\mathrm{x} \mathrm{H}^{2}$ (heredabilidad en el sentido amplio).

$\mathrm{GG}=35,5 \% \mathrm{x} 0,3=10,7 \%$ para el volumen si se utilizan los 10 individuos de la lista $\mathrm{A}$.

$\mathrm{GG}=33,7 \% \times 0,35=11,8 \%$ para calidad, con los árboles de la lista $\mathrm{A}$.

Si el árbol plus no se logra clonar, se procede a colectar su semilla. En este caso la estimación de su ganancia genética será inferior, debido que se utilizará la heredabilidad en el sentido estrecho.

$\mathrm{GG}=\mathrm{Sxh}^{2}=35,5 \mathrm{x} 0,25=8,87 \%$ para el volumen con los mejores 10 árboles de la lista $\mathrm{A}$.

$\mathrm{GG}=\mathrm{Sxh}^{2}=33,7 \times 0,30=10,1 \%$ para calidad, con $10 \mathrm{~s}$ árboles de la lista $\mathrm{A}$.

Estos valores de heredabilidad desde 0,25 hasta 0,35 para ambos caracteres, son realistas y conservadores, según se han reportado en programas de mejoramiento genético frecuentemente
(Cornelius 1994, Hodge y Dvorak 2003, Murillo y Badilla 2004). Los resultados de la estimación de ganancia genética por este método, suelen ser bajos debido a una subestimación del verdadero valor de diferencial de selección, tal y como se discutió anteriormente.

\section{CONCLUSIONES}

El método analítico propuesto en este trabajo, da un aporte novedoso y de gran base analítica a los programas de mejoramiento genético forestal. Permite orientar al mejorador en la definición de la población comercial (lista A) y la población de mejoramiento (lista $\mathrm{A}+\mathrm{B}$ ), tema fundamental para la estrategia de inicio de todo programa de mejoramiento genético. El método propuesto permite por primera vez, obtener un estimado del grado de progreso genético esperado del programa. Información vital para la toma de decisiones y la definición de estrategias de trabajo.

Es una herramienta que permitirá evaluar y validar la superioridad fenotípica de un árbol plus seleccionado, bajo un procedimiento analítico 
sustentable que consiste en un procedimiento que genera un registro y acredita el fenotipo de un árbol como superior. Permite clasificar a los árboles plus seleccionados en una lista A para utilización inmediata en la población comercial y una lista B para conformar parte de la población de mejoramiento y esperar los resultados de los ensayos genéticos. Permite también elaborar un ranking fenotípico de los árboles plus con base en su superioridad porcentual para cada carácter. Finalmente, podría utilizarse para estimar un valor porcentual de ganancia genética esperada, aunque con una tendencia a subestimar los verdaderos valores. La información que logra registrar de cada árbol seleccionado, puede ser de enorme utilidad a la hora de interpretar posteriormente los resultados de los ensayos genéticos.

\section{AGRADECIMIENTOS}

Un agradecimiento a la empresa BARCA S.A. por el apoyo en la realización de parte del estudio de campo incluido en este trabajo, así como en la utilización de información de su programa de mejoramiento genético. Este trabajo tuvo apoyo económico de la Vicerrectoría de Investigación y Extensión del Instituto Tecnológico de Costa Rica, mediante el financiamiento del proyecto de investigación "Mejoramiento genético forestal para sitios marginales: modelo de vinculación TEC-empresas forestales GENFORES".

\section{LITERATURA CITADA}

BADILLA Y., MURILLO O., OBANDO G. 2002. Efecto de la zona de vida y la altitud en la mortalidad y adaptabilidad al primer año de especies forestales en la Cordillera Volcánica Central, Costa Rica. Agronomía Costarricense 26(1):7-15.

BALCORTA MARTÍNEZ H.C., VARGAS HERNÁNDEZ J.J. 2004. Variación fenotípica y selección de árboles en una plantación de melina (Gmelina arborea Linn., Roxb.) de tres años de edad. Revista Chapingo, Serie ciencias forestales y del ambiente. 10(1):13-19.
CORNELIUS J. 1994. The effectiveness of plus-tree selection for yield. Forest Ecology and Management. (67):2334.

CRUZ COSME D. 2005. Princípios de Genética Quantitativa. Universidade Federal de Viçosa. Editora UFV. Viçosa, Minas Gerais, Brasil. 394 p.

GUTIÉRREZ B., QUINTERO P., NIETO V., MURILLO O. 2003. Enfoques cooperativos para el mejoramiento genético y la conservación de recursos genéticos forestales en Chile, Colombia y Costa Rica. Investigación Agraria: Sistemas y Recursos Forestales 12(3):111-122.

HODGE G.R., DVORAK W.S. 2003. The CAMCORE International Provenance/Progeny Trials of Gmelina arborea: genetic parameters and potential gain. In: W.S. Dvorak, G.R. Hodge, W.C. Woodbridge and J.L. Romero (eds.) Recent Advances with Gmelina arborea. CD-ROM. CAMCORE. USA. 20 p.

MURILLO O. 1991. Estrategias de Mejoramiento Genético Forestal, pp. 153-175. In: J.P Cornelius, J.F. Mesén y E.A. Corea (eds.). Manual sobre Mejoramiento Genético Forestal. CATIE. Costa Rica.

MURILLO O., BADILLA Y. 2004. Evaluación de la calidad y estimación del valor en pie de la plantación forestal. Escuela de Ingeniería Forestal, ITCR. Cartago, Costa Rica. 50 p.

MURILLO O., BADILLA Y., ARAYA E. 2004. Breeding teak in Costa Rica. In: IUFRO Meeting. Forest Genetics and Genomics. Consultado 1-5 de noviembre. Charleston, South Carolina, USA. www. ncsu.edu/feop/iufro_genetics2004/proceedings.pdf.

MURILLO O., OBANDO G., BADILLA Y., ARAYA E. 2001. Estrategia de mejoramiento genético para el Programa de Conservación y Mejoramiento Genético de especies forestales del ITCR/FUNDECOR, Costa Rica. Revista Forestal Latinoamericana 16 (30):273285 .

MURILLO O., ROJAS J.L., BADILLA Y. 2003. 2da edición. Reforestación Clonal. Taller de Publicaciones. Instituto Tecnológico de Costa Rica. Cartago, Costa Rica. 36 p.

RESENDE M.D.V., MURILLO O., BADILLA Y. En prensa. Genética Cuantitativa y Selección en el Mejoramiento Genético Forestal. Editorial Tecnológica de Costa Rica. Instituto Tecnológico de Costa Rica. Cartago, Costa Rica. 
TORRES J.M., MAGAÑA O. 2001. Evaluación de Plantaciones Forestales. Editorial Limusa, Noriega Editores. México, D.F., México. 472 p.

VALLEJOS J. 2007. Contribuciones al programa de mejoramiento genético de BARCA S.A. Informe de Práctica de Especialidad. Escuela de Ingeniería Forestal, Instituto Tecnológico de Costa Rica. Cartago, Costa Rica. 66 pp.

ZOBEL B., TALBERT J. 1984. Applied Forest Tree Improvement. John Wiley \& Sons. USA. 510 p. 